\title{
Anopbeles (A.) algeriensis Theobald, 1903 en Languedoc méditerranéen
}

\author{
Par Jean-A. RIOUX, Gilbert SINEGRE, Henri CROSET et André GABINEAU
}

Mentionné pour la première fois en France par J. Sautet (Provence, 1953), Anopheles (A.) algeriensis est actuellement connu de plusieurs localités de nos côtes tant méditerranéennes qu'atlantiques. Ainsi, en 1956, M. Sicart récoltait deux larves dans un émissaire de la Font-Estramer, résurgence vauclusienne des environs de Salses (Roussillon), tandis que R.-A. Heden (1957), puis J.-M. Doby, B. Rault et F. David (1960) signalaient à leur tour un chapelet de stations de l'Aunis à la Bretagne.

Jusqu'à présent, toutefois, l'espèce a surtout été récoltée en France sous sa forme larvaire et, par-là même, l'éthologie des adultes reste à préciser. Aussi bien, le but de cette courte note n'est pas de reprendre l'étude exhaustive de la question mais, plus modestement, d'apporter, outre quelques considérations écologiques et biogéographiques, une observation complémentaire sur le comportement trophique des imagos.

La nouvelle station d'Anopheles (A.) algeriensis, découverte le 6 août 1964 à l'occasion d'une prospection entomologique des lagunes du Narbonnais (Aude), est en réalité constituée par plusieurs gîtes égrenés dans le marais de la Capoulade, au lieu-dit \& Les Cabanes de Gruissan» (altitude 2 mètres, coordonnées 0,659 longitude Est de Paris, 47,91 latitude, carte 1/25.000 Narbonne 3-4).

Ultérieurement, cette même station a pu être revue et inventoriée les 14 octobre 1964, 4 décembre 1964, 11 janvier 1965, 26 février 1965, et 26 avril 1965.

\section{Ecologie}

Les gîtes larvaires, jouxtant le massif karztique de la Clape, s'étendent en ceinture sur plusieurs centaines de mètres le long de sa bordure occidentale. La nappe aquifère les alimente au contact de l'aptien moyen marneux, après avoir traversé les conglomérats oligocènes et les calcaires perméables de l'aptien supérieur. Les eaux viennent sourdre à travers les terrasses tyrrhéniennes et flandriennes (détritiques), dans le quaternaire récent limono-sableux, où sont localisés les gîtes (fig. 1). Une abondante végétation, composée essentiellement de pélophytes érigés, constitue un écran dense, masquant de manière à peu près totale la nappe aquatique (1).

(1) Nos observations concordent avec celles des auteurs soviétiques en Russie méridionale : «Les larves d'Anopheles algeriensis habitent les marécages alimentés par les eaux souterraines et ombragées par des roseaux et exigent une température entre 10 et $20^{\circ}$. $\gg$ (W. M. Beklemishev et A.N. Zhelokhovtzev, 1937). "Les larves d'Anopheles (A.) algeriensis ont été trouvées dans un ancien étang semi-artificiel $\left(600 \mathrm{~m}^{2}\right)$ alimenté par des sources... La surface de l'eau était entièrement recouverte d'une végétation haute et dense constituée de Phragmites communis, Scirpus lacustris, Alisma plantago...» (E. I. Lomeiko, 1942). 


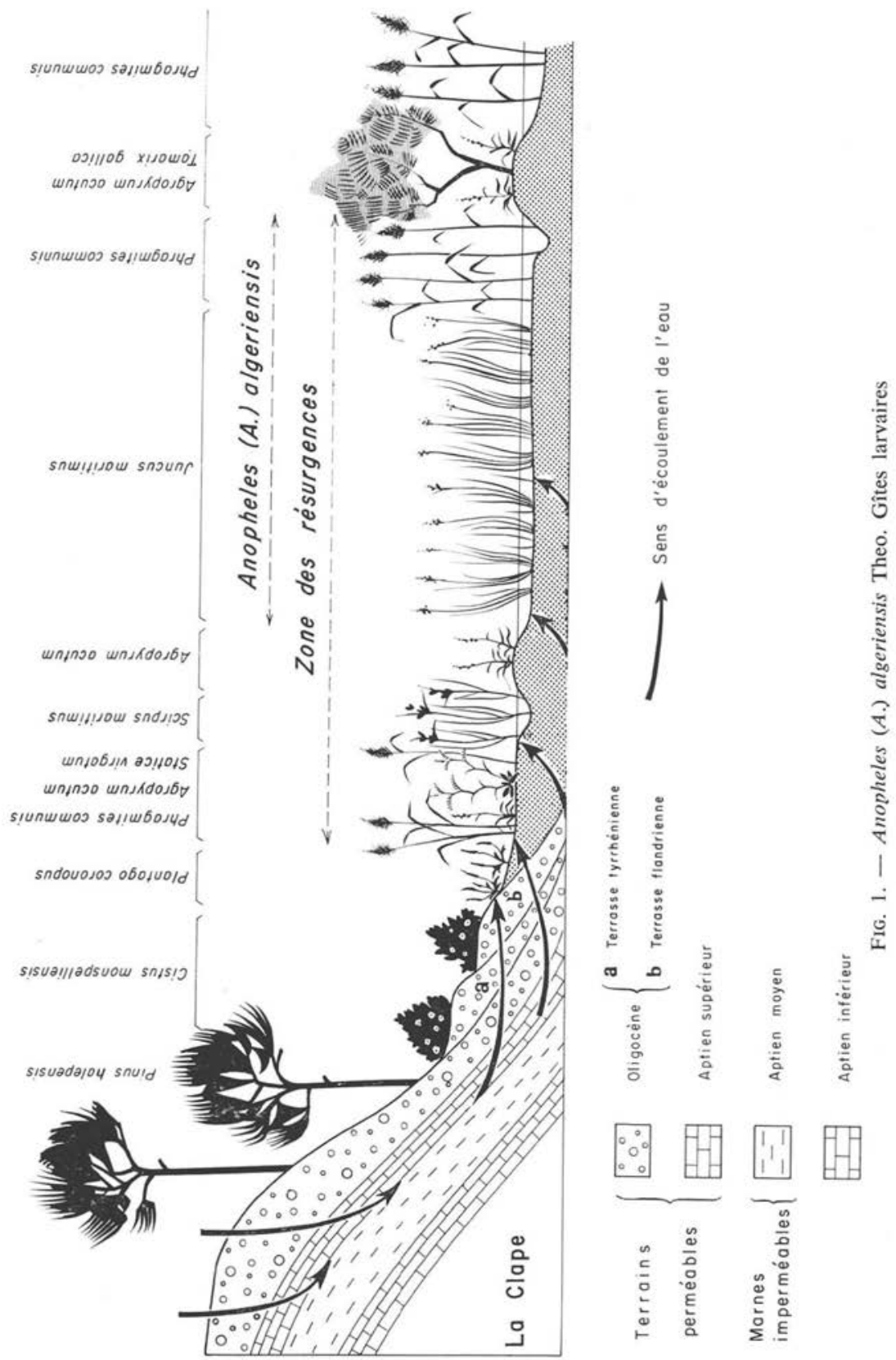


A première vue, le Phragmite (Phragmites communis Trin.) semble dominer largement. Toutefois, l'inventaire phytosociologique montre en réalité un mélange d'espèces semi-halophiles ou halophiles, parmi lesquelles le Jonc maritime (Juncus maritimus Lmk.) tient une place prépondérante (Junceto-triglochinetum maritimi $\mathrm{Br}$. Bl., 1931). Çà et là, ce dernier forme même des enclaves monospécifiques de quelques centaines de mètres, extrêmement denses, mais difficiles à repérer au sol en raison de l'écran vertical des Phragmites (fig. 2).

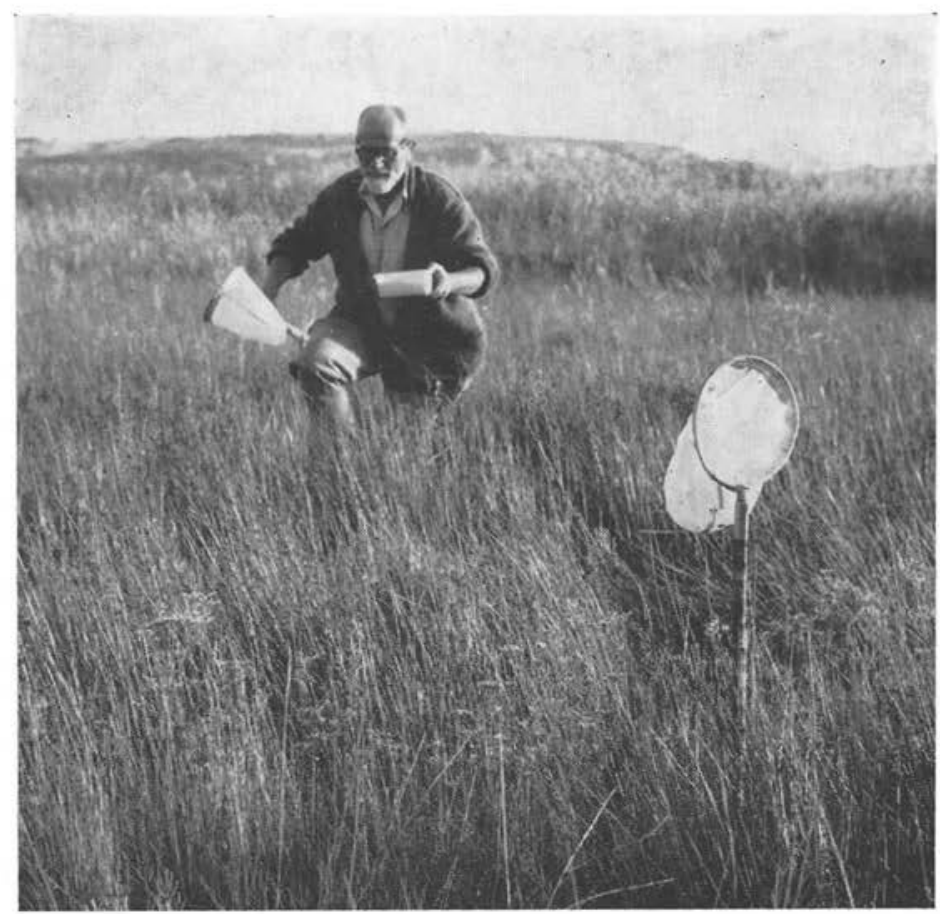

FIG. 2. - Gite à Anopheles (A.) algeriensis Theo. Les cabanes de Gruissan (Aude). Au premier plan, Salicorna fruticosa, Juncus maritimus et Statice limonium. A l'horizon, les collines de la Clape en partie masquée par un rideau de Phragmites communis.

Dans les zones drainées, la végétation s'ordonne en séries semi-linéaires, le long des rigoles; le fond du drain est en général occupé par le Jonc maritime, tandis que le sommet, habituellement exondé, abrite le grand Chiendent littoral (Agropyrum acutum Roem. et S.). Entre les deux espèces, s'observent, suivant la profondeur de la nappe et sa teneur en sel, tantôt le Triglochin (Triglochin maritimum L.), tantôt la Saladelle (Statice limonium L.), voire la Salicorne (Salicornia fruticosa L.). 


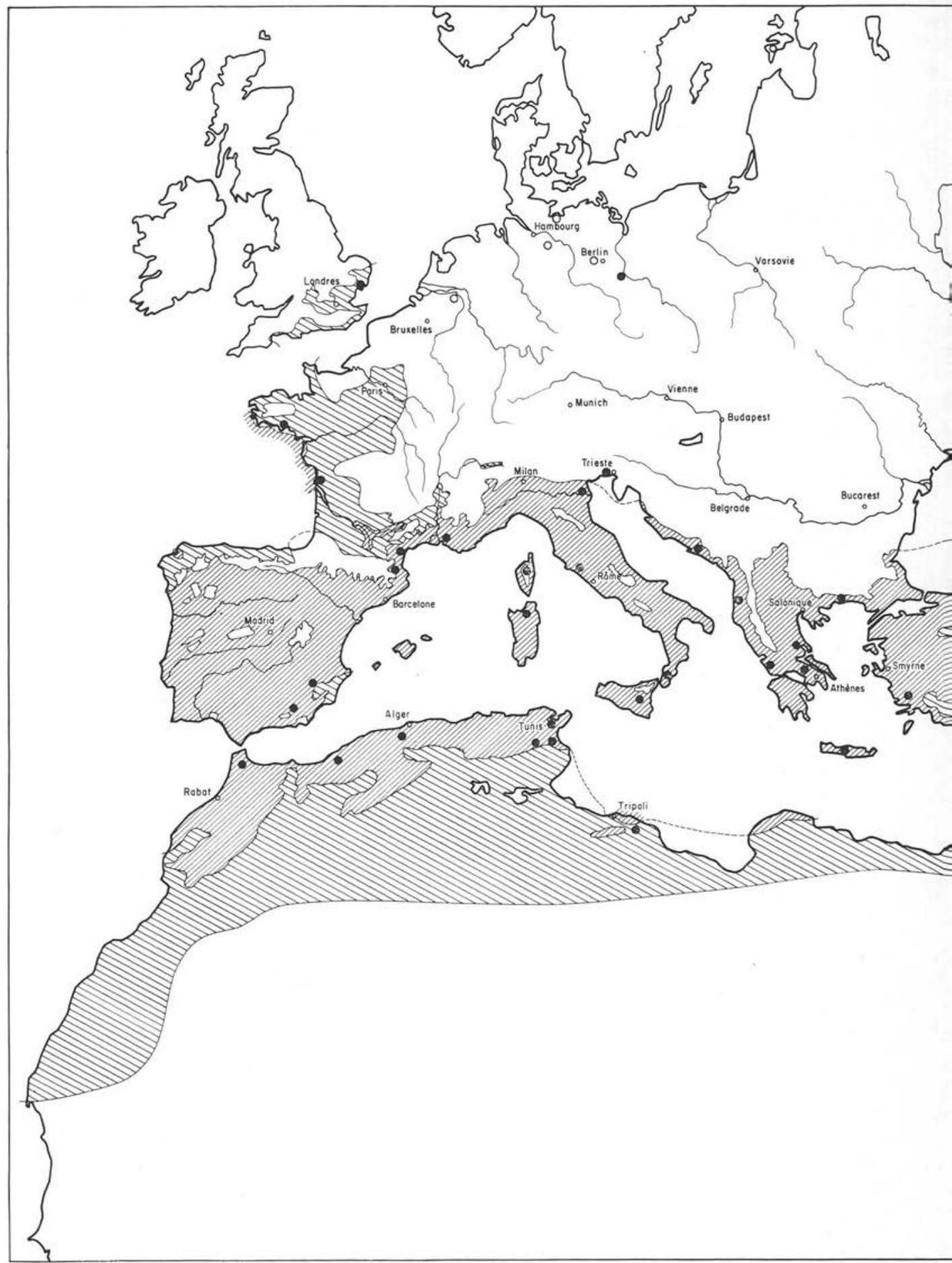

FIG. 3. - Répartition géograph 


\section{REPARTITION GEOGRAPHIQUE \\ d'Anopheles (A.) algeriensis}

\section{VIIIS Région méditerranéenne occidentale}

Région méditerranéenne orientale et latéméditerranéenne

- Localité d'A. algeriensis (larves adultes)

$\circ$ (odultes seuls)

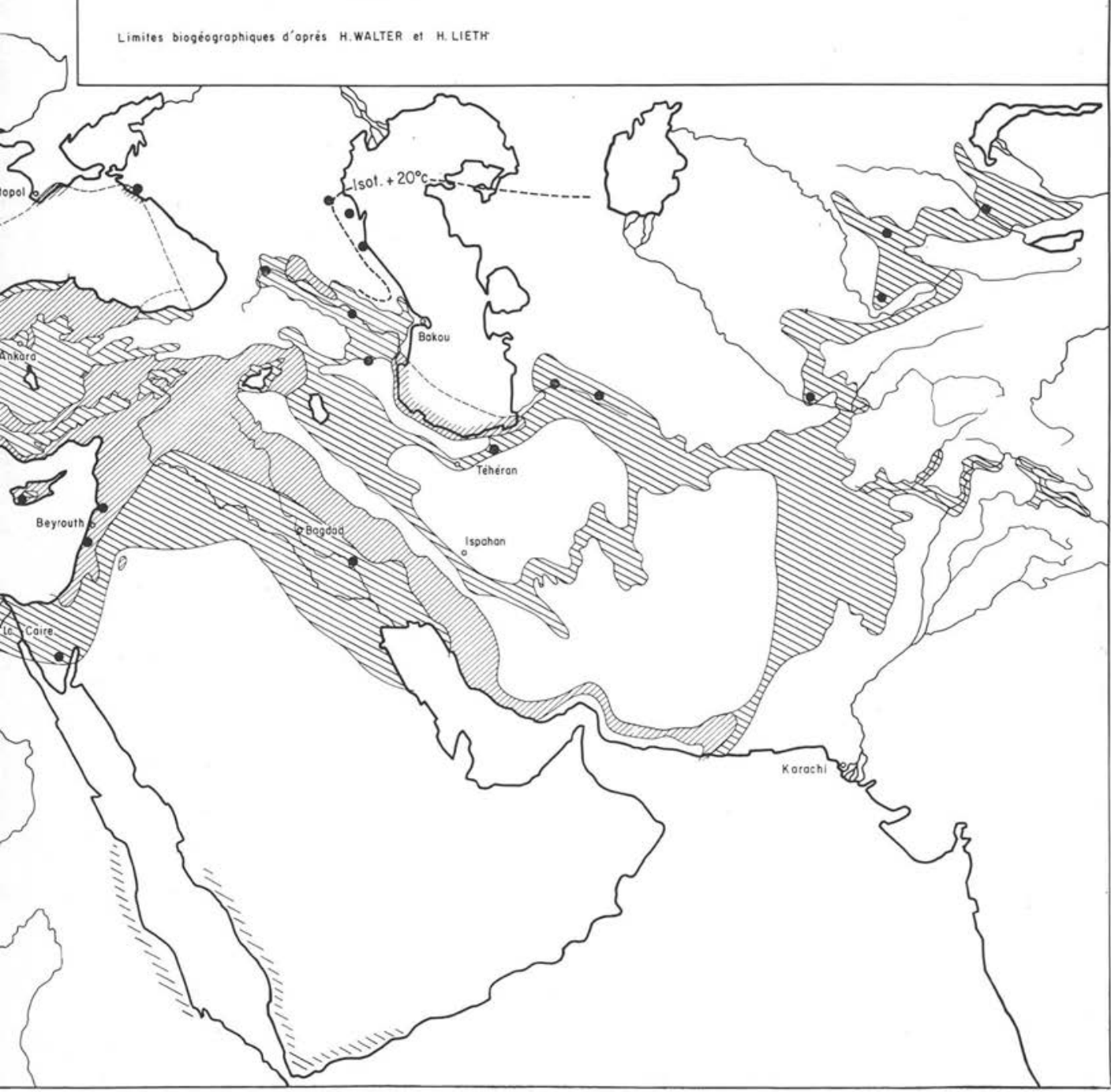

d'Anopheles (A.) algeriens Theo. 
L'analyse physico-chimique (cf. tableau) confirme d'ailleurs une assez forte minéralisation et explique également la présence, aux côtés d'Anopheles (A.) algeriensis, d'espèces halophiles [Aedes (O.) caspius, 14 octobre 1964] ou halobies [Aedes (O.) detritus, 3 décembre 1964].

\begin{tabular}{|c|c|c|c|c|c|}
\hline \multicolumn{6}{|c|}{ COMPOSANTE PHYTOSOCIOLOGIQUE (substrat limono-sableux) } \\
\hline & $\mathrm{N}^{\circ} 1$ & $\mathrm{~N}^{\circ} 2$ & $\mathrm{~N}^{\circ} 3$ & $\mathrm{~N}^{\circ} 4$ & Moyenne \\
\hline Juncus maritimus Lmk ..... & 4 & 4 & 3 & 5 & 4 \\
\hline Phrasmites communis Trin. ... & + & 2 & 3 & 1 & 2 \\
\hline Statice limonium L. ........ & 2 & . & . & $\therefore$ & \pm \\
\hline Aster tripolium L. ......... & 1 & $\therefore$ & . & $\therefore$ & $\therefore$ \\
\hline Juncus subulatus Forskal .... & . & $\therefore$ & $\dot{t}$ & . & $\therefore$ \\
\hline Agropyrum acutum Roem. et S. & 1 & . & . & . & $\therefore$ \\
\hline Plantago coronopus L. ...... & + & 2 & . & . & - \\
\hline Triglochim maritimum L. ... & $\mathrm{R}$ & + & $\mathrm{R}$ & . & $1-$ \\
\hline Salicornia fruticosa L. ...... & + & . & . & $\mathrm{R}$ & $\mathrm{R}$ \\
\hline Inula crithmoides L. ........ & $\therefore$ & R & . & . & $\mathrm{R}$ \\
\hline Obione portulacoides (L.) Moq. & $\therefore$ & . & . & . & $\mathrm{R}$ \\
\hline Sueda maritima (L.) Dumort. . & R & . & . & . & $\mathrm{R}$ \\
\hline \multicolumn{6}{|l|}{ COMPOSANTE PHYSICO-CHIMIQUE } \\
\hline $\mathrm{ClNa}(\mathrm{en} \mathrm{g} \%) \ldots \ldots \ldots \ldots$ & 18,9 & 7,15 & 9,27 & 8,50 & 10,95 \\
\hline $\mathrm{K}($ en $\mathrm{mg} \%) \ldots \ldots \ldots \ldots$ & 147 & 77 & 88 & 87 & 99,75 \\
\hline $\mathrm{Ca} \ldots \ldots \ldots \ldots \ldots$ & 300 & 190 & 215 & 190 & 223,75 \\
\hline $\mathrm{Mg} \ldots \ldots \ldots \ldots \ldots \ldots$ & 105 & 58 & 42 & 57 & 65,5 \\
\hline$p \mathrm{H} \quad \ldots \ldots \ldots \ldots \ldots \ldots \ldots \ldots \ldots$ & 6,8 & 6,6 & 6,8 & 6,6 & 6,7 \\
\hline $\mathrm{T}^{\circ}(16-18 \mathrm{~h}) \ldots \ldots \ldots \ldots \ldots$ & $13^{\circ} \mathrm{C}$ & $12^{\circ} \mathrm{C}$ & $13^{\circ} \mathrm{C}$ & $13^{\circ} \mathrm{C}$ & $12,75^{\circ} \mathrm{C}$ \\
\hline
\end{tabular}

\section{Ethologie}

La plupart des auteurs qui ont étudié le comportement d'Anopheles (A.) algeriensis ont insisté sur le caractère foncièrement exophile de l'espèce. Ainsi, dès 1933, F. Peus note une activité maximale à l'extérieur, après le coucher du soleil. En 1937, E. Enikolopov remarque également de nombreux vols en plein jour par temps couvert. D'après cet auteur, les femelles s'éloigneraient peu des gîtes larvaires, ce qui expliquerait pour une part le caractère discontinu des stations, mais aussi la pullulation locale de l'espèce.

$\mathrm{Au}$ cours de nos prospections, nous avons pu confirmer ces données. Ainsi, le 14 octobre, de 16 heures à 18 heures, par temps couvert, nous avons été attaqués 
massivement sur les gîtes larvaires, alors qu'à quelques centaines de mètres, dans le massif de la Clape, aucun adulte n'était capturé, ainsi d'ailleurs que dans les cabanes et les clapiers tout proches.

Dans les gîtes, Anopheles (A.) algeriensis, qui piquait en compagnie d'Aedes (O.) detritus et d'Aedes $(O$.) caspius, se reconnaissait facilement, outre ses caractères génériques, à sa teinte générale roussâtre, bien différente du noir d'Aedes $(O$.) detritus ou du jaune blanchâtre d'Aedes $(O$.) caspius.

L'hibernation d'Anopheles (A.) algeriensis en Bas-Languedoc paraît s'effectuer au stade larvaire (gîte en activité le 2 décembre, le 11 janvier et le 26 février). Un tel comportement (2) a d'ailleurs été observé en d'autres régions, en particulier en Angleterre (J.-F. Marshall, 1938) et en Russie caucasienne [E. Enikolopov, 1937 (3)]. Toutefois, plusieurs auteurs ont insisté sur la constance thermique des gîtes (M. Bates, W. N. Beklemischev et L. La Face). En France, M. Sicart a observé l'espèce dans une résurgence vauclusienne et, comme l'indique la structure géologique, il n'est pas interdit de penser que les gîtes du Narbonnais, au contact du massif karztique de la Clape, soient essentiellement alimentés par une eau profonde.

\section{Chorologie}

L'aire géographique d'Anopheles (A.) algeriensis s'étend largement sur l'ensemble de la région méditerranéenne, des piémonts occidentaux de l'Himalaya à l'Espagne, en passant par les plateaux irano-afghans, les contreforts du Caucase, le Proche-Orient, la Turquie, la Grèce, l'Albanie, la Yougoslavie, l'Italie, l'Arabie, l'Egypte et le Maghreb.

Par ailleurs, elle remonte vers le Nord, le long des côtes atlantiques, atteint l'Angleterre à Norfolk et égraine quelques stations en Allemagne septentrionale et en mer Baltique (fig. 3).

Le caractère assez aberrant de ces dernières stations mérite une courte discussion. La première en date, signalée par E. Martini en 1931, se rapporte à trois femelles capturées à l'intérieur d'une étable du Hinsbeck, près de Krefeld. Une ponte a pu être obtenue, que les * critères usuels » ont permis de rapporter à Anopheles algeriensis. Toutefois, de l'avis même de l'auteur, les œufs observés étaient différents de ceux qu'il avait décrits avec F. W. Edward en 1926.

En 1933, F. Peus décrit une nouvelle station dans la réserve de Schildorf, près de Berlin. Quatre ans plus tard, ce même auteur retrouve l'espèce aux environs du petit lac de Klöbig, à l'est de Berlin. Récemment (comm. écr.), une nouvelle localité vient d'être découverte dans l'est du Mecklembourg, près de Neustrelitz. Le Mecklembourg était d'ailleurs connu (F. Weyer, 1933) par une capture d'adultes près de Waren (comm. écr.). D'après ce dernier, un gîte larvaire (le premier connu en Alle-

(2) Dans l'optique d'une lutte insecticide essentiellement dirigée contre les stades larvaires, cette notion comporte un important corollaire logistique, à savoir la possibilité d'une atténuation rapide de la puissance par le traitement chimique hivernal.

(3) E. Enikopolov insiste sur la résistance de la larve au froid. Il a pu conserver des larves congelées pendant huit jours consécutifs. 
magne) aurait été découvert en 1958 par son élève Scherpner, dans un jardin au sud de Frankfort.

Quant à la station baltique, elle a été signalée par H.-J. Rem en 1957, en Estonie, dans l'île Saurema. Anopheles algeriensis attaquait l'homme en compagnie d'Anopheles claviger. Ici encore, les biotopes larvaires n'ont pu être détectés.

Au demeurant, l'existence de ces localités septentrionales, pour une espèce de répartition essentiellement méditerranéenne, n'est pas sans poser d'intéressants problèmes biogéographiques et systématiques. Dans ces stations, il serait en particulier intéressant d'étudier de façon plus précise la structure ovulaire, structure qui avait déjà intrigué E. Martini en 1933. Peut-être, à l'instar du complexe maculipennis et claviger, sommes-nous également en présence d'une espèce polymorphe. Du même coup seraient résolus les délicats problèmes de répartitions, actuellement sans réponse.

\section{Epidémiologie}

Un dernier point mérite enfin d'être discuté, celui de la responsabilité d'Anopheles (A.) algeriensis dans le cycle épidémiologique du paludisme autochtone.

A la suite des auteurs russes (4), nous pensons que le rôle de cette espèce peut être considéré comme négligeable sinon nul. La raison primordiale doit en être recherchée dans le caractère foncièrement exophile (5) d'Anopheles (A.) algeriensis, comportement qui, d'une part, diminue les contacts Moustique-Homme malade (6) et plus encore, aux limites de l'aire climatique des Plasmodium (et tel est le cas du Sud de la France), ne permet que très difficilement l'évolution complète du parasite (7).

\section{Bibliographie}

Artken (H. G.), 1954. - The Culicidae of Sardinia and Corsica. Bull. Entom. Res., 45 (3): 437-494.

BARber (M. A.) and RICE (J. B), 1935. - Malaria studies in Greece. The Malaria infection rate in Nature. Ann. Trop. Med., 29:329-348.

(4) En Russie du Sud, les paludologues insistent sur l'absence de corrélation entre les foyers de paludisme et les zones à Anopheles (A.) algeriensis : "Apparemment, dans la transmission du paludisme, le rôle d'Anopheles (A.) algeriensis est insignifiant car les ouvriers habitant ces régions marécageuses, si intensément peuplées par cette espèce d'Anophèles, ne souffrent que très peu du paludisme. Au contraire, les habitants des villages voisins où pullulent $A$. maculipennis souffrent énormément de cette maladie.» (S. K Enikolopov, 1937).

(5) C'est vraisemblablement pour cette même raison qu'Anopheles (A.) hyrcanus [dont le larve est assez souvent associée à celle d'Anopheles (A.) algeriensis, M. Bates, N. Beklemishev, E. Lomeiko, L. La Face, 1949], abondante en Camargue, et en Languedoc, ne joue également aucun rôle dans l'épidémiologie du paludisme autochtone.

(6) Par l'identification du sang ingéré, M. A. Barber et J. B. Rice (Grèce, 1933) ont obtenu un faible pourcentage pour Anopheles (A.) algeriensis (16\%) par rapport à Anopheles (A.) sacharovi $(61 \%)$.

(7) Ainsi, le paludisme de réimportation des années 1943-1946 a disparu rapidement de la vallée de Liri (Italie), en partie grâce à l'effet irritant du D.D.T. qui contraignait les Anophèles vecteurs normalement endophiles, à gagner des gîtes de repos extérieurs où les conditions thermo-clima. tiques ne permettaient plus l'évolution normale du cycle sporogonique (M. Coluzzi). 
Beklemishev (W. M.) et Zhelokhovtev (A. N.), 1937. - Les aires de plusieurs espèces d'Anophèles de l'U.R.S.S. et les facteurs dont elles dépendent. Bull. Soc. Nat. Biol. Moscou, $50(1-2): 71-73$.

Boyd (M. F.), 1949. - Malariology. Saunders Co. ed., T. I, 787 p.

Buxton (P. A.), 1923. - A Key to the fourth stage Anopheles larvae of Palestine. Bull. Soc. Roy. Ent. d'Egypte, 45-50.

CuBoni (E.), 1926. - Osservazioni sulla biologia dell "Anopheles superpictus » et dell' «Anopheles algeriensis» e caratteri differenziali delle loro larve. Riv. di Mal., 1: 49-54.

Doby (J.-M.), Rault (B.), David (F.), 1960. - Theobaldia (Culicella) litorea (Shute 1928) et Anopheles (A.) algeriensis Theo. 1903, dans l'Ouest de la France. Contribution à l'étude écologique de ces deux espèces. Ann. Paras. Hum. Comp., 35 (1-2): 174187.

Edwards (F. W.), 1928, -- The Nematocerous Diptera of Corsica-Diptera Lechevalier éd., 4 (4) : $157-189$

Emberger (L.), 1942. - Un projet d'une classification des climats du point de vue phytogéographique. Bull. Soc. Hist. Nat. Toulouse, $77:$ 97-124.

—, 1943. - Les limites de l'aire de végétation méditerranéenne en France. Bull. Soc. Hist. Nat. Toulouse, $78: 159-180$.

-, 1952. - Phytogéographie. Sur le quctient pluviothermique. C.R. Soc. Acad. Sciences, $227: 2508-2510$.

Enikolopov (S. K.), 1937. - Au sujet de l'écologie des Anopheles algeriensis Theo. 1903. Med. Paras. and Paras. Diseases, 6 (3) : 354-359.

Guillaume (A.), 1960. - La zone méditerranéenne en France (III' partie), Bull. Soc. Bot. France, $107(7-8): 273-290$.

Hart (G. F. W.), 1954. - Rediscovery of Anopheles algeriensis Theo. (Dipt. Culicidae) in Norfolk. Ent. Mon. Mag., $171: 63$.

HEdeEn (R. A.), 1957. - A note on the occurrence of Anopheles algeriensis Theo. in Western France. Mosq. News, 17: 97.

LA FACE (L.), 1926. - Revisione degli Anofelini Italiani. Note 1, Riv. di Mal., 5 (1) : 44-49.

Lissova (A. I.), 1935. - Contribut:on à la connaissance de la faune des moustiques de la famille Culicidae de la ville de Tashkent et des environs. Mag. Paras., Inst. Zool. Acad. Sc. Léningrad, $5: 27-54$.

LomeIкo (E. I.), 1942. - Anopheles algeriensis Theo. dans la province autonome d'Adyghey, dans le Nord du Caucase. Med. Paras. and Paras. Dis., 11: 131.

Marshall (J. F.), 1938. - British mosquitoes. British Museum (Nat. Hist.), 341 p.

MARTINI (E.), 1931. - Ueber Anopheles bifurcatus und algeriensis. Anzeiger für Schädlingskunde, 7: 109-110.

Mattingly (P. F.), 1950. - Handbooks for the identification of British Insects. Roy. Ent. Soc. London, 9 (2) : 102-120.

Peus (F.), 1933, - Herr Peus berichtet über neuere Culiciden. Mitt. der Deut. Ent. Gesellschaft, $4: 10-145$.

Peus (F.), 1937. - Ein neuer Fundort von Anopheles algeriensis Theo. in der Mark. Mitt. der Deut. Ent. Gesel., 7 (6-7): 68. 
REM (H. J.), 1957. - Zur Faunistik und ökologie der Culiciden (Diptera, Culicidae) der Estnischen U.R.S.S. Rev. Ent. de l'U.R.S.S., 36 (l) : 148-160.

Rioux (J. A.), 1958. - Les Culicides du «Midi» méditerranéen. Enc. Ent. Lechevalier, éd., $303 \mathrm{p}$.

SAlem (H. H.), M. B., Ch. B., M. Sc., D. T. M. and H., 1938. - The mosquito Fauna of Sinaï Peninsula (Egypt) with a description of two new species. Bull. Soc. Roy. Ent. d'Egypte, 16 : 1-47.

Sautet (J.), 1953. - Les fluctuations du paludisme dans le Sud de la France et dans les départements insulaires. Rev. Path. Gén. Comp., 652: 1199-1203.

SÊnevet (G.) et Andarelli (L.), 1956. - Les Anophèles de l'Afrique du Nord et du Bassin méditerranéen, Lechevalier éd., $280 \mathrm{p}$.

SiCART (M.), 1956. - Sur la présence d'Anopheles algeriensis Theo. en Roussillon. Montp. Med., 49 (5) : 480-481.

Stone (A.), 1959. - A synoptic catalog of the Mosquitoes of the World. Soc. Ent. of America, ed., 358 p.

Stone (A.), 1961. - A synoptic catalog of the Mosquitoes of the World. Supplement I

StONE (A.), 1959. - A synoptic catalog of the Mosquitoes of the World. Supplement I (Diptera: Culicidae). Ent. Soc. Washington, 1:29-52.

Toumanoff (C.), 1954. - Contribution à l'étude de l'Anophélisme et du Paludisme en Corse. Monographie de l'I.N.H., 113 p.

Vecchio (G. DEL), 1940. - Osservazioni sull' Anopheles algeriensis. Riv. di Paras. 4 (4): 221-225,

Walter (H.) und Lieth (H.), 1964. - Klimadiagram Weltatlas, V.E.B., G. Fischer éd.

WeYER (F.), 1959. - Uber schwankungen in der zusammensetzung Natürlicher Anopheles populationen in Deutschland. Riv. di Paras. 20 (4) : 435-444.

[Ecologie médicale et Pathologie parasitaire, Faculté de médecine, Montpellier (Hérault)] 